Rev. Adm. Saúde Vol. 17, Nº6, Jan. - Mar. 2017

ARTIGO ORIGINAL

\title{
Parto normal ou cesárea: a influência do tipo de parto desejado na satisfação materna quanto à assistência hospitalar ao nascimento
}

Normal delivery or cesarean section: the influence of desired type of birth on maternal satisfaction with hospital care for childbirth

\section{Angela Maria Bacha ${ }^{1}$, Oswaldo da Rocha Grassiotto ${ }^{2}$, Denis Barbosa Cacique ${ }^{3}$, Helymar da Costa Machado ${ }^{4}$, Gislaine Aparecida Fonsechi-Carvasan ${ }^{5}$, Claudia Aparecida Batista de Souza ${ }^{6}$}

\author{
1 Doutora em Tocoginecologia pela Unicamp; Docente do Departamento de Tocoginecologia da \\ FCM/Unicamp - Campinas (SP), Brasil
}

2 Doutor em Tocoginecologia pela Unicamp; Vice-Reitor Executivo de Administração/Unicamp - Campinas (SP), Brasil

3 Bacharel em Filosofia e Mestre em Ciências da Saúde pela Unicamp; Assistente da Diretoria do Hospital da Mulher-Professor Dr. José Aristodemo Pinotti Caism/Unicamp - Campinas (SP), Brasil

4 Graduado em Estatística pela Universidade Federal de São Carlos e Mestre em Estatística pela Unicamp; Estatístico do Hospital da Mulher-Professor Dr. José Aristodemo Pinotti Caism/Unicamp - Campinas (SP), Brasil

5 Graduada em Estatística e Mestra em Saúde Coletiva pela Unicamp; Estatística do Hospital da MulherProfessor Dr. José Aristodemo Pinotti Caism/Unicamp - Campinas (SP), Brasil

6 Graduanda em Pedagogia (Licenciatura) pela Faculdade de Educação e bolsista do programa de Bolsa Auxílio Social do Serviço de Apoio ao Estudante/Unicamp - Campinas (SP), Brasil

\section{RESUMO}

O estudo objetivou avaliar a satisfação materna quanto à assistência hospitalar ao parto considerando-se o tipo de parto desejado e o realizado. Tratou-se de estudo descritivo, de corte transversal, com abordagem quantitativa e amostragem por conveniência em um hospital de ensino, em Campinas, São Paulo. 181 puérperas responderam, durante a internação, questionário contendo 18 questões. Pacientes internadas por mais dias apresentavam maior insatisfação para todos os domínios avaliados. Pacientes com menor nível socioeconômico estavam mais insatisfeitas com o parto. No comparativo entre parto desejado e realizado, os grupos mais insatisfeitos eram os de mulheres cuja via de parto desejada foi diferente da realizada: mulheres que desejavam um parto normal e tiveram 
uma cesariana foram as mais insatisfeitas. Conclui-se que as diferenças de satisfação em relação à via de parto são estatisticamente significativas apenas quando se inclui na análise tanto o desejo da paciente como a via efetiva de parto. Face à problemática da cesariana no Brasil esse estudo contribui para uma melhor compreensão do excesso de cesarianas praticados no país bem como ajuda na busca de soluções adequadas para esse problema.

Palavras-chave: Satisfação do Paciente. Cesárea. Parto Normal. Parto Desejado. Alojamento Conjunto. Tempo de Internação.

\begin{abstract}
The purpose of this study was to evaluate maternal satisfaction with hospital care for childbirth and postpartum hospitalization, considering the relationship between the desired and performed type of delivery. It was conducted a descriptive and cross-sectional study, with quantitative approach and convenience sampling, in a University hospital, in Campinas, São Paulo State. 181 women answered an interview during the stay in the rooming-in, based on a closed questionnaire containing 18 questions. Patients hospitalized for more days had greater dissatisfaction for all domains assessed. Patients with lower socioeconomic status were more dissatisfied with delivery than those with higher socioeconomic level. In the comparison between desired and performed type of delivery, the most dissatisfied group consisted of women whose desired and performed type of delivery didn't match each other: women who wanted a natural birth and had a Csection were the most dissatisfied. We concluded that the differences of satisfaction according to the type of delivery are statistically significant only when the analysis includes both the desired and the performed type of delivery. This study contributes to a better understanding of excess cesareans in Brazil as well as can help in finding appropriate solutions to this problem.
\end{abstract}

Keywords: Patient Satisfaction; Cesarean Section; Natural Childbirth; Desired Birth; Rooming-in Care; Length of Stay.

\title{
INTRODUÇÃO
}

Gravidez, parto e puerpério constituem a principal causa de internação nos serviços públicos e privados de saúde brasileiros ${ }^{1}$. Nesse cenário, grande atenção deve ser dada à avaliação da satisfação das pacientes, iniciativa considerada um aspecto importante da humanização do parto ${ }^{2}$. Dar voz à mulher, permitindo-lhe manifestar-se sobre os pontos positivos e negativos da assistência, vai ao encontro do tão necessário reconhecimento do protagonismo da parturiente nesse momento marcante da vida. Além disso, conforme ressalta Donabedian, informações sobre a satisfação de pacientes são indispensáveis tanto para a avaliação da qualidade da assistência como para o próprio desenvolvimento e administração de serviços de saúde ${ }^{3}$.

Parte dos esforços dedicados à avaliação da satisfação sobre a assistência ao parto e ao 
pós-parto reside na investigação do conjunto de características que influenciariam a satisfação das pacientes. Alguns dos temas mais frequentemente incluídos nesse tipo de análise são a participação da gestante em diferentes programas de pré-natal, a qualidade da comunicação com a equipe de saúde, o papel da enfermagem durante o cuidado e o tempo de permanência na internação pós-parto ${ }^{4}$. Outros trabalhos têm procurado estabelecer relação entre a satisfação da parturiente e os métodos farmacológicos empregados para o alívio da dor ${ }^{5}$. E identificam-se, ainda, estudos voltados à avaliação da influência de características pessoais das mulheres sobre a satisfação, como idade, raça, escolaridade, estado civil, paridade e nível socioeconômico ${ }^{6}$.

Nesse conjunto de determinantes, destaca-se a relação entre satisfação e via de parto. Estudo realizado na Austrália, por exemplo, identificou diferenças estatisticamente significativas de satisfação entre os partos do tipo vaginal espontâneo (com a maior satisfação), cesárea eletiva (segunda maior), cesárea de emergência (terceira) e vaginal instrumental (quarta) ${ }^{7}$. Na Irlanda, estudo concluiu que pacientes que haviam realizado parto vaginal (com cesárea prévia) estavam mais satisfeitas do que aquelas que haviam realizado parto cesárea (com parto normal prévio) em relação às informações relacionadas ao pré-parto e ao parto em $\mathrm{si}^{8}$. Já uma pesquisa nacional verificou que a satisfação média para os dois tipos de partos era mesma, salientando-se que as pacientes que haviam realizado cesáreas estavam mais satisfeitas com o manejo da dor pós-parto, ao passo que aquelas que tinham realizado parto vaginal estavam mais satisfeitas com o controle da dor durante o trabalho de parto ${ }^{9}$.

O estudo da relação entre via de parto e satisfação das parturientes deve ter destacada relevância no Brasil, cuja taxa de cesáreas foi reconhecida pelo Fundo das Nações Unidas para a Infância como uma das mais elevadas do mundo ${ }^{10}$. No país, dependendo do município e do tipo de financiamento analisados, a proporção de cesarianas supera $90 \%$ dos partos, com crescimento constante ao longo dos últimos anos ${ }^{11}$. O temor materno em relação à dor e ao trabalho de parto, a preocupação parental com o bemestar fetal, eventuais experiências negativas com partos anteriores e a conveniência de se agendar o parto são alguns dos motivos apontados como determinantes da alta incidência de cesarianas ${ }^{12}$. No centro dessa discussão, portanto, juntamente com outros fatores, está a preferência da mulher por determinada via de parto. Todavia, embora existam estudos que procurem identificar e enumerar os componentes dessa preferência ${ }^{13}$, bem como outros que enfatizem a importância da participação da mulher nas decisões referentes ao parto ${ }^{4}$, não se encontram na literatura pesquisas que relacionem a satisfação da puérpera com as vias de parto desejado e realizado.

Diante dessas considerações, o objetivo do presente estudo consistiu em avaliar a satisfação de puérperas em relação ao parto e à internação pós-parto em um hospital situado na região metropolitana de Campinas, São Paulo. Como objetivo específico, o estudo pretendeu comparar os níveis de satisfação entre mulheres que haviam realizado parto vaginal e cesariano. Também foram comparados os níveis de satisfação segundo 0 tipo de parto desejado (normal ou cesárea), verificando, ainda, se haveria diferença de satisfação caso o parto efetivamente realizado não fosse igual àquele desejado pela paciente.

\section{MÉTODOS}

A pesquisa foi realizada no ano de 2014, em um hospital de ensino, público, que atende exclusivamente pelo SUS e que é referência para o acompanhamento da gravidez e do 
parto de alto risco. A instituição integra a Rede Cegonha e detém certificação da Iniciativa Hospital Amigo da Criança, estimulando, dentre outros cuidados, o incentivo ao aleitamento materno logo após o nascimento, a presença de acompanhante (antes, durante e após o parto) e a utilização seletiva e não rotineira de procedimentos invasivos (como, por exemplo, a episiotomia). O serviço realiza aproximadamente 2.800 partos por ano, para os quais dispõe de 15 leitos de terapia intensiva e 15 de cuidados intermediários neonatais, 6 de UTI adulto, 20 de internação clínica durante a gravidez e 24 de puerpério em sistema de alojamento conjunto.

Tratou-se de estudo descritivo, de corte transversal, com abordagem quantitativa e amostragem por conveniência. $O$ cálculo do número de sujeitos utilizou técnica de amostragem para estimação de proporção de população finita ${ }^{14}$, assumindo-se para $p$ (proporção de pacientes satisfeitas) o valor de 0,5. A população considerada para esse cálculo consistiu na média trimestral de internações na enfermaria do Alojamento Conjunto (557 pacientes). O erro amostral foi de $6 \%$ e o nível de significância igual a 5\%. Com isso, o tamanho amostral foi de 181 sujeitos.

A coleta de dados foi realizada diariamente por entrevistadores nas Enfermarias de Alojamento Conjunto, unidade de internação que admite mulheres em pós-parto, que estejam amamentando e cujos recém-nascidos possam permanecer com elas. Podiam participar do estudo puérperas de qualquer idade, com pelo menos 24 horas de internação no Alojamento Conjunto, que não estivessem recebendo atendimento médico ou da enfermagem no momento da entrevista e que consentissem com a participação no estudo. O projeto foi aprovado pelo Comitê de Ética em Pesquisa da Faculdade de Ciências Médicas da Unicamp (Parecer no 496/2011), que dispensou a utilização do Termo de Consentimento Livre e Esclarecido após entendimento de que a identificação das participantes poderia inibir o ingresso no estudo ou comprometer a veracidade das informações fornecidas.

O questionário utilizado nas entrevistas possui 18 questões, respondidas mediante uma escala do tipo Likert com os seguintes pesos: "ruim"=1, "médio"=2, "bom"=3 e "excelente" $=4$. O escore de satisfação de cada participante foi obtido somando-se os valores das respostas e dividindo-se o resultado pelo número de questões respondidas, o que gerou uma média. A seguir, essa média foi convertida em uma escala de 0 a 100 pontos, de tal modo que, quanto maior fosse o escore, melhor seria a satisfação. A transformação da média na escala de 0 a 100 pontos utilizou a seguinte fórmula: escore $=($ média dos itens -1$) /(4-1) \times 100$. Para o escore total, foram utilizadas as 18 questões, ao passo que, para os domínios, a média foi calculada de acordo com a composição de cada um deles.

As questões foram agrupadas em quatro domínios: "Pronto Atendimento", "Parto", "Relacionamento Interpessoal" e "Organização do Serviço". O primeiro deles inclui questões sobre a admissão da paciente via Pronto Atendimento, como o tempo de espera e a educação do médico e do enfermeiro. O domínio "Parto" aborda aspectos como a presença de acompanhante durante o parto e os procedimentos realizados para aliviar a dor. O "Relacionamento Interpessoal" trata de questões diretamente relacionadas à interação com os profissionais da saúde, como a clareza das orientações e a delicadeza da equipe no cuidado da puérpera e do recém-nascido. Finalmente, a "Organização do Serviço" aborda aspectos físicos do local de internação (como temperatura do quarto e conforto da cama) e características relacionadas à organização do serviço (como o respeito à privacidade, limpeza e nível de ruídos). A composição desses domínios inspirou-se no modelo teórico de Donabedian, que estabelece um conjunto de categorias 
("structure", "process" e "outcome") para avaliar a qualidade do cuidado na área da saúde ${ }^{3}$.

Além das questões diretamente relacionadas à satisfação das pacientes, foram coletados dados sobre o tempo de internação (em dias), idade (em anos), número de gestações, escolaridade, estado civil, tipo de parto realizado (vaginal ou cesárea) e tipo de parto inicialmente desejado pela paciente (normal ou cesárea). A classificação socioeconômica baseou-se na pontuação estabelecida pelo Critério de Classificação Econômica Brasil da Associação Brasileira de Empresas de Pesquisa ${ }^{15}$.

Os dados coletados foram submetidos ao teste de Shapiro-Wilk ${ }^{16}$, verificando-se que a distribuição dos escores de satisfação não era normal. A descrição da amostra foi realizada por meio de tabelas de frequência e do cálculo de estatísticas descritivas: média, desvio padrão, mediana e valores mínimo e máximo. A comparação entre os níveis de satisfação e as variáveis categóricas empregou o teste de Mann-Whitney (para variáveis com dois grupos) e o teste de Kruskal-Wallis (para variáveis com três ou mais categorias) $)^{17}$.

Quanto ao questionário empregado, para testar a hipótese da composição fatorial da escala de satisfação das pacientes em quatro domínios, utilizou-se análise fatorial confirmatória, via modelo de equações estruturais para variáveis latentes ${ }^{18}$. A consistência interna geral e por domínio foi avaliada por meio do coeficiente alfa de Cronbach, considerando-se que o questionário teria boa consistência para resultados maiores que $0,70^{19,20}$. A validação concorrente (ou "de critério") do escore geral e de cada um de seus domínios foi avaliada correlacionando-os com uma questão respondida à parte pelas pacientes ("como você avalia a internação como um todo"). Para isso, utilizou-se o coeficiente de correlação de Spearman, considerando-se que valores elevados indicariam uma validação concorrente satisfatória ${ }^{21}$. Todos os testes estatísticos foram realizados por meio do software SAS versão 9.2, adotando-se o nível de significância de 5\%, ou seja, $\mathrm{P}<0,05$.

\section{RESULTADOS}

A caracterização socioeconômica das participantes consta da Tabela 1. A amostra constituiu-se de 181 mulheres, com idade média de 25,5 anos. Em relação à escolaridade, pouco mais da metade delas possuíam pelo menos o nível médio completo.

Tabela 1. Caracterização da amostra segundo idade, escolaridade, estado civil, classificação socioeconômica, tipo de parto realizado e desejado, presença de acompanhante, planejamento da gravidez, números de gestações e de filhos e tempo de internação.

\begin{tabular}{l|l}
\hline Variáveis & $\mathrm{n}(\%)$ \\
\hline Idade (anos) & $25,5(7,2)$ \\
Média (desvio padrão) & $24,0(14,0 ; 44,0)$
\end{tabular}


Escolaridade

Analfabeto / Fundamental I incompleto

$1(0,6)$

Fundamental I completo / Fundamental II incompleto

$31(17,1)$

Fundamental II completo / Médio incompleto

$56(30,9)$

Médio completo / Superior incompleto

$83(45,9)$

Superior completo

$10(5,5)$

Estado civil

Solteira

Casada ou mora com o companheiro $145(80,1)$

Divorciada

$4(2,2)$

Critério Brasil/Classificação socioeconômica

D-E (pobres e extremamente pobres)

C2 (vulneráveis) $46(25,6)$

C1 (baixa classe média) $69(38,3)$

B2 (média classe média) $43(24,0)$

B1 (alta classe média)

A (classe alta)

Parto realizado

Vaginal

$115(63,5)$

Cesárea

$66(36,5)$

Parto desejado

Normal

$134(74,0)$

Cesárea

$33(18,2)$

Indiferente

$14(7,8)$

Presença de acompanhante durante o parto

Sim

Não

Número de gestações

Primigesta

$80(44,2)$ 
Duração da internação (dias)

Média (desvio padrão)

Mediana (mínimo; máximo)

$2,0(1,0 ; 14,0)$

NOTA: Amostra total de 181 mulheres. Uma participante não respondeu as questões para classificação socioeconômica.

A maioria era casada ou morava com o companheiro. E quase $75 \%$ da amostra se concentravam entre as classes $\mathrm{C}, \mathrm{D}$ e $\mathrm{E}$. O tempo médio de internação no momento da entrevista era de 2,6 dias. A maioria das mulheres (74,0\%) desejava ter realizado um parto normal, enquanto $18,2 \%$ preferiam a cesariana e as demais não tinham preferência. Quanto aos partos efetivamente realizados, $63,5 \%$ foram vaginais e $36,5 \%$ cesarianos. A maioria das participantes contou com a presença de acompanhantes durante o parto, com $81,2 \%$ da amostra.

Os dados sobre a validade concorrente, estrutura fatorial, consistência interna do questionário e escores de satisfação por questão e por domínio constam da Tabela 2. A validação concorrente obteve valores satisfatórios para o escore geral do questionário e para os domínios "Parto", "Relacionamento Interpessoal" e "Organização do Serviço". Todas as questões obtiveram carga fatorial maior que 0,30 para seus respectivos domínios, sempre com significância estatística. A consistência interna obteve valores acima de 0,70 para os quatro domínios do instrumento, assim como para o escore geral.

Tabela 2. Questionário de avaliação da satisfação das pacientes internadas no Alojamento Conjunto, segundo composição dos domínios, cargas fatoriais de cada item, coeficiente alfa de Cronbach, validação concorrente e escores de satisfação geral, por domínio e por questão.

\begin{tabular}{|c|c|c|c|c|c|c|}
\hline Domínios e respectivos itens avaliados & \begin{tabular}{|l|} 
Carga \\
Fatorial
\end{tabular} & $\begin{array}{l}\text { Valor } \\
\text { de t }\end{array}$ & $\begin{array}{l}\text { Alfa de } \\
\text { Cronbach }\end{array}$ & $\begin{array}{l}\text { Validação } \\
\text { Concorren } \\
\text { te ** }\end{array}$ & \begin{tabular}{|l} 
Escor \\
e \\
Médi \\
0
\end{tabular} & $\begin{array}{l}\text { Desvio } \\
\text { Padrão }\end{array}$ \\
\hline Pronto Atendimento & & & 0,728 & 0,555 & 68,7 & 21,4 \\
\hline $\begin{array}{l}\text { Atendimento na recepção do Pronto } \\
\text { Atendimento }\end{array}$ & 0.654 & $7.52^{*}$ & & & 67,6 & 25,6 \\
\hline $\begin{array}{l}\text { Educação do médico e do enfermeiro do } \\
\text { PA }\end{array}$ & 0.849 & $10.41^{*}$ & & & 79,8 & 20,9 \\
\hline $\begin{array}{l}\text { Tempo de espera no Pronto Atendimento } \\
\text { para a internação }\end{array}$ & 0.579 & $6.48^{*}$ & & & 58,5 & 33,2 \\
\hline Parto & & & 0,726 & 0,629 & 81,6 & 19,1 \\
\hline Ajuda dos profissionais durante o parto & 0.724 & $8.46^{*}$ & & & 82,4 & 23,0 \\
\hline
\end{tabular}


Presença de acompanhante durante o parto

Procedimentos realizados para aliviar a dor durante o parto
$0.7929 .45^{*}$

$83,5 \quad 20,8$

$77,2 \quad 28,2$

\begin{tabular}{|c|c|c|c|c|c|c|}
\hline Relacionamento Interpessoal & & & 0,894 & 0,638 & 84,1 & 16,2 \\
\hline $\begin{array}{l}\text { Educação dos profissionais do Alojamento } \\
\text { Conjunto }\end{array}$ & 0.775 & $10.04^{*}$ & & & 84,4 & 18,1 \\
\hline $\begin{array}{l}\text { Clareza das informações recebidas sobre } \\
\text { a sua saúde }\end{array}$ & 0.716 & $8.98^{*}$ & & & 82,0 & 21,5 \\
\hline $\begin{array}{l}\text { Orientações sobre como amamentar, dar } \\
\text { banho e segurar o bebê }\end{array}$ & 0.858 & $11.73^{*}$ & & & 86,5 & 17,2 \\
\hline $\begin{array}{l}\text { Delicadeza com que médicos e } \\
\text { enfermeiros cuidam de você e do bebê }\end{array}$ & 0.919 & $13.15^{\star}$ & & & 86,9 & 17,8 \\
\hline Agilidade do atendimento no hospital & 0.712 & $8.91^{*}$ & & & 81,2 & 22,3 \\
\hline Organização do Serviço & & & 0,894 & 0,690 & 75,8 & 16,2 \\
\hline Alimentação servida a você & 0.694 & $8.57^{*}$ & & & 78,8 & 21,9 \\
\hline $\begin{array}{l}\text { Limpeza do hospital (chão, roupas, lençol, } \\
\text { banheiro e corredores) }\end{array}$ & 0.668 & $8.14^{*}$ & & & 78,1 & 21,2 \\
\hline $\begin{array}{l}\text { Conforto da cama (colchão, travesseiro, } \\
\text { lençol e cobertor) }\end{array}$ & 0.827 & $11.01^{*}$ & & & 80,5 & 20,2 \\
\hline Temperatura do quarto & 0.362 & $4.00^{*}$ & & & 57,1 & 32,5 \\
\hline Respeito a sua privacidade & 0.778 & $10.04^{*}$ & & & 79,8 & 20,4 \\
\hline Presença de barulhos & 0.712 & $8.88^{*}$ & & & 74,2 & 26,5 \\
\hline Segurança dentro do hospital & 0.826 & $10.99^{*}$ & & & 82,4 & 17,8 \\
\hline Escore Geral do Questionário & & & 0,935 & 0,742 & 77,9 & 14,9 \\
\hline $\begin{array}{l}\text { Questão extra: como você avalia a } \\
\text { internação como um todo? }\end{array}$ & & & & & 82,1 & 19,1 \\
\hline
\end{tabular}

NOTA: Todos os itens se iniciavam com a questão "Como você avalia a/o..." e podiam ser respondidos mediante a seguinte escala do tipo Likert: ruim, médio, bom e excelente.

* Valores significativos das cargas padronizadas dos itens $(p<0.05)$ para valor de $t>1.96$, segundo análise fatorial confirmatória.

** Coeficiente de correlação de Spearman.

Com relação aos escores de satisfação por questão e domínio, o "Relacionamento Interpessoal" e o "Parto" obtiveram as melhores avaliações, ambos com escores acima de 80 pontos. Já o "Pronto Atendimento" obteve a pior avaliação, seguido pela "Organização do Serviço". A satisfação geral foi de 77,9 pontos e a questão extra sobre como a paciente avaliava a internação como um todo obteve 82,1 pontos. Aprofundando-se a análise para examinar individualmente cada questão, verifica-se que os três aspectos 
mais bem avaliados estavam diretamente ligados à relação entre as mulheres entrevistadas e os profissionais da saúde, incluindo a delicadeza de médicos e enfermeiros, as orientações recebidas e a educação da equipe no Alojamento Conjunto. Dentre os itens menos bem avaliados, dois estavam relacionados ao Pronto Atendimento, incluindo o tempo de espera para internação e o atendimento na recepção. O aspecto com pior avaliação foi a temperatura do quarto no Alojamento Conjunto.

O tempo de internação influenciou a satisfação das pacientes, conforme Tabela 3. De modo geral, houve uma redução expressiva da satisfação a partir do terceiro dia de internação, quer em relação ao segundo dia, quer em relação ao primeiro. Nos domínios "Pronto Atendimento" e "Parto", a diminuição da satisfação é significativa no comparativo entre o primeiro e o terceiro dias de internação. Já em "Relacionamento Interpessoal" e "Organização do Serviço", nota-se uma redução significativa da satisfação na passagem do segundo para o terceiro dia. Essa tendência também se observa em relação à satisfação geral das pacientes, com diferença estatisticamente significativa de 7,4 pontos entre o segundo e o terceiro dia de internação.

Tabela 3. Satisfação das pacientes em relação ao tempo de internação no momento da entrevista.

\begin{tabular}{l|l|l|l|l}
\hline \multirow{2}{*}{ Domínios Avaliados } & 1 dia & 2 dias & $\geq 3$ dias & Valor-P \\
\cline { 2 - 5 } & $n=57$ & $n=53$ & $n=71$ & \\
\hline Pronto Atendimento & & & \multicolumn{2}{c}{0,020} \\
Média (desvio padrão) & $73,3(19,3)$ & $71,2(20,3)$ & $62,9(22,8)$ \\
Mediana & $77,8^{*}$ & 66,7 & $66,7^{*}$ \\
Mínimo; Máximo & $(22,2 ; 100,0)$ & $(22,2 ; 100,0)$ & $(0,0 ; 100,0)$
\end{tabular}

Parto

Média (desvio padrão)

$85,2(16,2) \quad 84,7(18,7) \quad 76,3(20,5)$

Mediana

$88,9^{*}$

88,9

$66,7^{*}$

Mínimo; Máximo

$(33,3 ; 100,0)$

$(22,2 ; 100,0)$

$(11,1 ; 100,0)$

Relacionamento Interpessoal

Média (desvio padrão)

$85,2(16,3) \quad 87,6(14,6) \quad 80,7(16,8)$

Mediana

93,3

$93,3^{*}$

$80,0^{*}$

Mínimo; Máximo

$(40,0 ; 100,0) \quad(60,0 ; 100,0)$

$(46,7 ; 100,0)$

\section{Organização do Serviço}

Média (desvio padrão)

$76,2(14,5) \quad 80,1(14,7) \quad 72,3(17,9)$

Mediana

71,4

$85,7^{*}$

$66,7^{*}$ 
Mínimo; Máximo

$(47,6 ; 100,0) \quad(42,9 ; 100,0)$

$(33,3 ; 100,0)$

Satisfação Geral

Média (desvio padrão)

$79,6(13,8) \quad 81,4(12,9) \quad 74,0(16,3)$

Mediana

79,6

$85,2^{*}$

$70,4^{*}$

Mínimo; Máximo

$(47,1 ; 100,0)$

$(53,7 ; 100,0)$

$(46,3 ; 98,2)$

NOTA: Valor-P referente ao teste de Kruskal-Wallis. ${ }^{*}$ Grupos com diferença significativa $(\mathrm{P}<0,05)$ pelo teste post-hoc de Dunn.

A classificação socioeconômica apresentou relação estatisticamente significativa com a satisfação das pacientes no domínio "Parto". Conforme Tabela 4, observa-se que as pacientes pobres e extremamente pobres estavam mais insatisfeitas do que aquelas situadas nos estratos entre a baixa classe média e a classe alta. Para os demais domínios, não foram observadas diferenças de satisfação.

Tabela 4. Satisfação das pacientes segundo a classificação socioeconômica.

\begin{tabular}{|c|c|c|c|c|c|}
\hline \multirow{3}{*}{ Domínio } & \multicolumn{4}{|c|}{ Classificação Socioeconômica (Critério Brasil) } & \multirow{3}{*}{ Valor-P } \\
\hline & D-E & $\mathrm{C} 2$ & C1 & B-A & \\
\hline & $n=19$ & $n=46$ & $\mathrm{n}=69$ & $\mathrm{n}=46$ & \\
\hline \multicolumn{5}{|l|}{ Parto } & \multirow[t]{4}{*}{0,028} \\
\hline Média (desvio padrão) & $70,8(19,4)$ & $81,3(16,5)$ & $85,2(18,2)$ & $81,2(21,5)$ & \\
\hline Mediana & $66,7^{*}$ & 83,3 & $88,9^{*}$ & $88,9^{*}$ & \\
\hline Mínimo; Máximo & $(44,4 ; 100,0)$ & $(33,3 ; 100,0)$ & $\begin{array}{l}(11,1 ; \\
100,0)\end{array}$ & $\begin{array}{l}(22,2 ; \\
100,0)\end{array}$ & \\
\hline
\end{tabular}

Não foram identificadas diferenças estatisticamente significativas de satisfação no comparativo entre os tipos de parto realizado, fossem eles normais, cesarianos ou com utilização de fórceps. Também não se identificaram diferenças significativas de satisfação entre pacientes que desejavam parto do tipo normal ou cesariano. Ou seja, isoladamente, tanto o tipo de parto realizado como o tipo de parto desejado não afetaram a satisfação das pacientes. Entretanto, quando o tipo de parto realizado foi relacionado com o tipo de parto desejado, observou-se diferença significativa de satisfação, conforme Tabela 5.

Tabela 5. Satisfação das pacientes no comparativo entre o tipo de parto desejado e o 
efetivamente realizado.

\begin{tabular}{|c|c|c|c|c|c|}
\hline \multirow[t]{2}{*}{ Domínios Avaliados } & \begin{tabular}{|l|} 
Desejou e \\
Realizou \\
parto Normal
\end{tabular} & $\begin{array}{l}\text { Desejou e } \\
\text { Realizou } \\
\text { parto } \\
\text { Cesariano }\end{array}$ & $\begin{array}{l}\text { Desejou parto } \\
\text { Normal e } \\
\text { realizou } \\
\text { Cesariano }\end{array}$ & $\begin{array}{l}\text { Desejou } \\
\text { Cesariano e } \\
\text { Realizou } \\
\text { Normal }\end{array}$ & \multirow[t]{2}{*}{ Valor P } \\
\hline & $\mathrm{n}=98$ & $n=23$ & $n=33$ & $\mathrm{n}=8$ & \\
\hline \multicolumn{5}{|l|}{ Parto } & \multirow[t]{4}{*}{0,043} \\
\hline Média (desvio padrão) & $83,0(19,9)$ & $86,2(16,7)$ & $75,3(16,3)$ & $75,0(20,1)$ & \\
\hline Mediana & 88,9 & $100,0^{*}$ & $66,7^{*}$ & $75,0^{*}$ & \\
\hline Mínimo; Máximo & $(11,1 ; 100,0)$ & $(55,6 ; 100,0)$ & $(44,4 ; 100,0)$ & $(44,4 ; 100,0)$ & \\
\hline
\end{tabular}

\section{Relacionamento Interpessoal}

0,025

$\begin{array}{lllll}\text { Média (desvio padrão) } & 85,8(15,2) & 89,6(14,0) & 79,6(16,2) & 75,8(17,4) \\ \text { Mediana } & 93,3 & 100,0^{*} & 66,7^{*} & 66,7^{*} \\ \text { Mínimo; Máximo } & (53,3 ; 100,0) & (66,7 ; 100,0) & (60,0 ; 100,0) & (53,3 ; 100,0)\end{array}$

\section{Total Geral}

Média (desvio padrão)

$79,2(13,7)$

$82,5(13,9)$

$72,8(16,6)$

$71,7(14,9)$

Mediana

79,6

$86,3^{*}$

$66,7^{*}$

$66,8^{*}$

Mínimo; Máximo

$(51,9 ; 100,0)$

$(57,4 ; 100,0)$

$(48,2 ; 100,0)$

$(55,6 ; 96,3)$

Valor-P referente ao teste de Kruskal-Wallis. * Grupos com diferença significativa $(P<0,05)$ pelo teste post-hoc de Dunn. Foram excluídas da comparação 5 mulheres que realizaram parto vaginal com utilização de fórceps e 14 mulheres que disseram não ter preferência em relação ao tipo de parto que viria a ser realizado.

Nos domínios "Parto" e "Relacionamento Interpessoal", bem como no escore geral, pacientes que desejavam e realizaram parto cesariano estavam mais satisfeitas do que aquelas que desejavam cesariana e realizaram parto normal, ou que desejavam parto normal e realizaram cesariana. Semelhantemente, pacientes que desejavam e realizaram parto normal também apresentaram escores elevados de satisfação, sem, no entanto, diferença estatisticamente significativa na comparação com os demais grupos.

Quando a amostra é agrupada em duas categorias, de acordo com o atendimento ou não da expectativa da mulher sobre a via de parto, observa-se que os maiores graus de satisfação concentram-se no grupo cujos partos se deram pela via desejada pelas pacientes, fosse ela vaginal ou cesariana, conforme gráficos da Figura 1. Essa diferença é estatisticamente significativa para os domínios "Parto" $(p=0,005)$, "Relacionamento Interpessoal" ( $p=0,007)$ e "Organização do Serviço" $(p=0,011)$, bem como para o escore geral de satisfação $(p=0,008)$. 
Figura 1. Gráficos comparativos dos escores de satisfação de pacientes do Alojamento Conjunto entre tipo de parto desejado e realizado, excluindo fórceps e indiferentes.

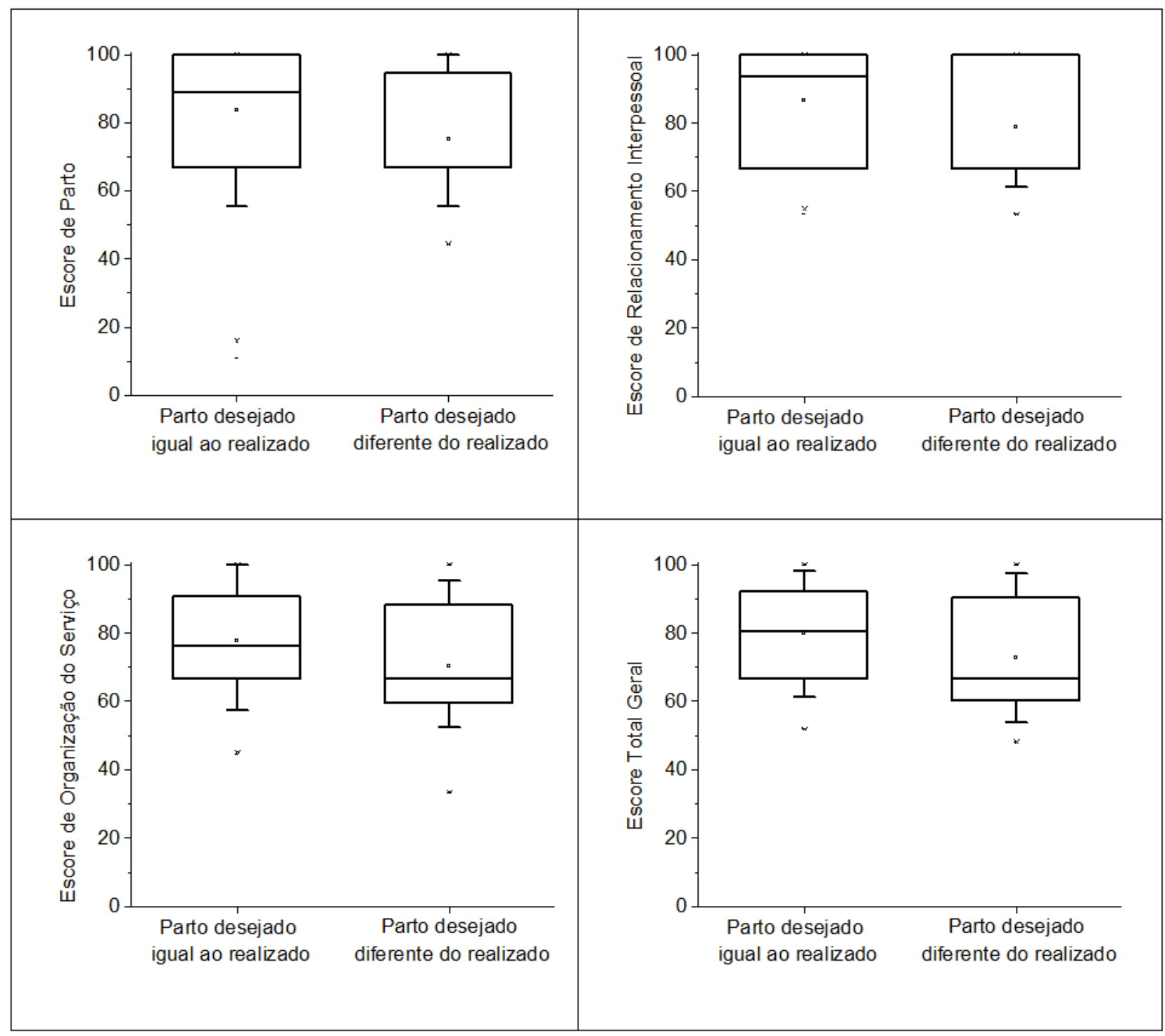

Não apresentaram relação estatisticamente significativa com a satisfação as variáveis sobre presença de acompanhante durante o parto, número de gestações, idade, estado civil e escolaridade, cujos dados não são apresentados.

\section{DISCUSSÃO}

O presente estudo verificou que os maiores graus de satisfação foram obtidos pelo domínio "Relacionamento Interpessoal", que aborda, principalmente, os cuidados e orientações ofertados por médicos e enfermeiros durante a internação da paciente no Alojamento Conjunto, portanto, após o parto. Nesse momento, mãe e bebê saudáveis permanecem juntos um ao outro; a amamentação é estimulada, a presença do 
acompanhante é frequente e o binômio recebe a visita de outros familiares. Em contrapartida, o domínio "Pronto Atendimento" obteve os piores escores de satisfação, principalmente na avaliação do tempo de espera e do atendimento na recepção do serviço. Pode-se supor que a diferença entre esses dois domínios esteja relacionada à ansiedade e a um possível desconforto físico da parturiente num momento anterior ao seu acolhimento no hospital, prejudicando sua satisfação. É oportuno mencionar que o evento do parto está frequentemente associado a um sentimento de ansiedade intensa ${ }^{22,23}$. Além disso, a percepção e as expectativas das pacientes sobre o tempo de espera em serviços de emergência são muito mais importantes na determinação da satisfação do que o tempo efetivo de espera ${ }^{24}$. À medida, porém, que a gestante é acolhida pela equipe do hospital, tem seu parto realizado de forma humanizada e pode, enfim, permanecer junto de seu filho e amamentá-lo, observa-se um aumento da satisfação.

No domínio "Parto", a questão sobre os procedimentos realizados para aliviar a dor obteve escore de satisfação inferior àquelas quanto à presença de acompanhantes e à ajuda de profissionais. A literatura tem observado que a relação entre satisfação, dor de parto e analgesia é complexa. Embora o fenômeno da dor possa influenciar a satisfação das pacientes, essa relação não é óbvia, direta, ou sequer tão forte quanto a influência que os profissionais da saúde podem exercer por meio de suas atitudes ${ }^{5}$. De qualquer modo, é salutar a realização de novos estudos que permitam ampliar o conhecimento sobre a relação entre dor no parto e satisfação, sobretudo explorando a influência de métodos farmacológicos e não farmacológicos para o controle da dor.

Outros aspectos que apresentaram maior insatisfação estavam relacionados, principalmente, ao ambiente físico e à organização do Alojamento Conjunto. A presença de barulhos, por exemplo, obteve uma das piores avaliações de todo o questionário, provavelmente em virtude de a unidade, na época deste estudo, possuir quartos grandes (contendo de três a oito leitos), com internação tanto da mãe como do recém-nascido e recebendo, ainda, a visita controlada de familiares, além da circulação de médicos, enfermeiros e demais profissionais responsáveis pelo cuidado das pacientes. $\mathrm{O}$ que a literatura tem demonstrado é que pacientes internadas em quartos individuais detém maior satisfação do que aquelas admitidas em quartos coletivos ${ }^{25}$. Essa diferença afeta não apenas os aspectos físicos do ambiente, mas também o cuidado da enfermagem, as orientações recebidas sobre o cuidado com o bebê, a amamentação e o plano de alta.

Um dos fatores identificados como determinantes da satisfação foi o tempo de internação, com tendência de diminuição da satisfação à medida que as pacientes permaneciam internadas por mais dias. Esse resultado é condizente com um estudo que comparou a satisfação de dois grupos de pacientes que haviam realizado cesárea de emergência. $O$ primeiro deles, com maior satisfação, incluiu pacientes com baixa permanência (3,6 dias), enquanto o segundo, mais insatisfeito, incluiu pacientes com permanência normal $(4,8$ dias) ${ }^{26}$. Outra pesquisa concluiu que $97 \%$ das pacientes recomendariam a suas amigas um programa de internação pós-parto de curta duração, 93\% participariam dele novamente e $93 \%$ consideraram seu tempo de internação adequado ${ }^{27}$. Entretanto, não são apresentados dados sobre a satisfação das pacientes, verificando se haveria diferenças de satisfação à medida que o tempo de internação avançasse ${ }^{27}$.

Um dos desafios associados à redução do tempo de internação de pacientes em pósparto é a implementação de um programa efetivo de cuidado domiciliar (ou de outro modelo de fácil acesso à puérpera), viabilizando a promoção de qualquer intervenção que, de outro modo, seria ofertada no interior da maternidade ${ }^{4}$. A pesquisa da fenilcetonúria, por exemplo, popularmente conhecida como "teste do pezinho", tem como 
objetivo detectar e tratar precocemente doenças que, se prevenidas, evitam sequelas como a deficiência mental ${ }^{28}$. Entretanto, dado que as concentrações de fenilalanina podem não estar significativamente elevadas até que o lactente tenha ingerido proteína dietética durante as primeiras 48 horas de vida, a realização desse teste não deve ocorrer precocemente $^{29}$. Segue-se que algumas mães podem permanecer internadas por mais tempo do que julgam ser necessário, tendo em vista a disponibilidade de horários nos quais o teste da fenilalanina pode ser realizado no hospital.

O nível socioeconômico foi outro fator a relacionar-se com a satisfação das pacientes, com menores graus de satisfação no domínio "Parto" para as pacientes pobres e extremamente pobres, no comparativo com aquelas situadas nos estratos mais favorecidos. Esse resultado é condizente com a revisão de literatura de Sitzia e Wood, segundo a qual, pacientes mais pobres detêm menor satisfação ${ }^{30}$. Esse dado, porém, é considerado pouco consistente pelos autores, dada a dificuldade de se mensurar as variáveis socioeconômicas. De qualquer modo, outra revisão internacional também verificou que as pacientes mais favorecidas recebem melhor tratamento dos médicos, ainda que se considere o mesmo serviço de saúde ${ }^{31}$. No cenário brasileiro, estudo de coorte de base hospitalar com 15.688 mulheres concluiu que as maiores chances de satisfação geral para com a atenção ao parto estavam associadas a mulheres pertencentes às classes $A$ e $B$, enquanto aquelas de cor da pele preta ou parda tinham a menor chance de estarem satisfeitas ${ }^{32}$. De acordo com os autores, existiria em alguns serviços públicos do país uma cultura discriminatória, marcada pela concepção de que as mulheres de baixa renda e de pouca escolaridade não teriam capacidade de entendimento para decidir sobre as intervenções no parto. Segue-se, de acordo com o referido estudo, que essas mulheres relatam menor clareza nas explicações, pouca abertura a eventuais perguntas e reduzida participação nas decisões.

No que diz respeito à relação entre o tipo de parto realizado e a satisfação das parturientes, não foram identificadas diferenças estatisticamente significativas de satisfação entre as cesarianas e os partos vaginais, resultado semelhante ao de outro estudo nacional publicado recentemente ${ }^{33}$. Por outro lado, se observaram diferenças estatisticamente significativas de satisfação na relação entre o tipo de parto realizado e desejado, sendo que os menores graus de satisfação foram identificados entre as pacientes com expectativas frustradas em relação à via efetiva do parto. Com efeito, a literatura tem referido que a satisfação materna é influenciada pela expectativa das mulheres, nem sempre retratando a qualidade real do atendimento ${ }^{32}$. Entretanto, muitos trabalhos não apresentam uma distinção clara entre os conceitos de expectativa e preferência em relação ao parto: enquanto o primeiro está usualmente baseado no conhecimento do que é possível, o segundo concerne ao desejo da mulher ${ }^{5}$. Nesse sentido, cumpre ressaltar que o grupo mais insatisfeito do presente estudo, no comparativo entre tipos de parto desejado e realizado, era composto de oito mulheres que desejavam realizar cesariana, mas evoluíram para parto vaginal. Portanto, embora não possuíssem indicação médica para a realização da cirurgia cesariana, elas desejavam fazê-lo e sentiram-se insatisfeitas com a frustração dessa expectativa.

A preferência da mulher por determinada via de parto é um dos elementos mais frequentemente apontados como responsáveis pela alta prevalência de cesarianas ${ }^{13}$. Estudo nacional recente verificou que 39,3\% das mulheres nulíparas e 29,5\% das primíparas tinham preferência pela cesariana ${ }^{33}$. No presente estudo, 33 mulheres manifestaram o desejo por esse tipo de parto, o que corresponde a 18,2\% da amostra. Ainda que pese a escolha da mulher, cumpre frisar que a cesariana é uma alternativa médica idealmente utilizada em situações em que as condições materno-fetais não 
favorecem o parto vaginal ${ }^{34}$. Além disso, embora se deva reconhecer a segurança da cesárea moderna e as contribuições dessa intervenção para uma melhor assistência à saúde, é importante que sua indicação seja criteriosa, pois não se trata de um procedimento inócuo e sua realização pode trazer riscos adicionais para a mãe e a criança $^{34}$.

Portanto, conforme recomendou um estudo brasileiro, cabe aos profissionais que atuam na assistência pré-natal avaliar as razões implícitas na demanda da mulher pela cesariana, discutindo os riscos e benefícios dessa cirurgia em relação ao parto vaginal ${ }^{13}$. Além de proporcionar maior segurança à saúde dos envolvidos, essa estratégia tende a resultar em maior satisfação da paciente, pois, de acordo com a literatura, decisões referentes a procedimentos médicos e a medicações tendem a ser mais bem aceitas pela mulher e seu acompanhante quando eles compreendem as razões implícitas nessas decisões ${ }^{4}$.

A discussão dessa temática, porém, não deve se restringir ao contexto hospitalar de atenção ao parto ou de acompanhamento da gravidez. Os benefícios do parto normal em comparação à cesariana, bem como os riscos frequentemente ignorados na preferência por este procedimento cirúrgico, necessitam ser divulgados amplamente na sociedade brasileira, em caráter educativo e em benefício da saúde pública, de modo a modificar a chamada "cultura da cesariana". A abordagem desse tema em escolas, universidades e centros comunitários, por exemplo, certamente contribuiria para a desmistificação de uma série de temores relacionados ao parto vaginal. Além disso, é crucial que esse tema seja tratado de forma responsável em filmes, novelas e séries de televisão, promovendo uma cultura que privilegie a escolha daquela - parto normal - que é reconhecidamente a melhor via de parto para conclusão da gestação, em detrimento daquela - cesariana - que se propagandeia, direta ou indiretamente, como a mais cômoda, fácil ou adequada a determinadas classes sociais.

Com relação às limitações do presente estudo, cabe mencionar que foram incluídas apenas mulheres em pós-parto internadas em sistema de alojamento conjunto, portanto, que pudessem amamentar e cujos recém-nascidos não necessitassem de terapia intensiva ou semi-intensiva neonatal. É salutar que, futuramente, seja promovida a avaliação da satisfação baseada em amostras mais heterogêneas do ponto de vista da saúde da mulher e do recém-nascido, a fim de corroborar os resultados identificados no presente estudo.

Também é necessário considerar um possível efeito do viés de gratidão. Problema recorrente na avaliação da satisfação em serviços públicos de saúde, esse viés caracteriza-se pela omissão de questionamentos e críticas negativas dos usuários, que temem ter o acesso à assistência prejudicado como resultado da participação no estudo ${ }^{35}$. Essa dificuldade tende a ser ainda maior no caso da assistência perinatal, pois as puérperas podem se sentir aliviadas, agradecidas e com sentimentos positivos depois do nascimento de uma criança saudável, "compensando qualquer experiência negativa da assistência"6. Para reduzir a influência desse viés, no presente estudo não foram coletados quaisquer dados que permitissem a identificação da paciente (como nome, número de matrícula ou de quaisquer outros documentos pessoais), de modo a assegurar o sigilo quanto às opiniões emitidas.

\section{CONCLUSÕES}


Os resultados do presente estudo permitem concluir que a satisfação das puérperas quanto ao atendimento no Pronto Atendimento tende a ser inferior em relação aos demais setores em que ocorre 0 acolhimento à mulher que busca assistência hospitalar para 0 parto, como o Alojamento Conjunto e o Centro Obstétrico, por exemplo. Em contrapartida, os maiores graus de satisfação se observam na relação estabelecida com os profissionais da saúde na internação posterior ao parto, quando mãe e recém-nascidos encontram-se juntos em sistema de alojamento conjunto, sendo possível a amamentação e o recebimento de visitas.

Com relação aos determinantes da satisfação, pode-se concluir que o tempo de internação, o nível socioeconômico das pacientes e a relação entre tipo de parto desejado e realizado desempenham papéis importantes, influenciando a satisfação para com diferentes aspectos da atenção ao parto e da internação pós-parto. O tempo de internação está inversamente relacionado com a satisfação sobre todos os aspectos da assistência: pacientes internadas há mais tempo apresentam menores graus de satisfação quanto ao Pronto Atendimento, ao parto, à relação com a equipe de saúde e com a organização do serviço. Já o nível socioeconômico das puérperas influencia a satisfação quanto ao parto, de tal modo que pacientes pobres e extremamente pobres detêm menor satisfação do que aquelas oriundas da média classe média e da classe alta.

Finalmente, o presente estudo permite concluir que as diferenças de satisfação em relação à via de parto são estatisticamente significativas apenas quando se inclui na análise o desejo prévio da paciente quanto a essa via. O que se observa é que as pacientes com expectativas frustradas detém menor satisfação especificamente quanto ao parto e à relação com os profissionais que a assistem. Nessa comparação, o grupo mais insatisfeito é o de mulheres com desejo de realizar cesariana, mas com indicação e realização efetiva de parto vaginal. Por outro lado, as pacientes que desejavam e realizaram parto vaginal (as quais representavam o maior grupo nesse comparativo) apresentaram altos níveis de satisfação, semelhantes aos das pacientes que queriam e realizaram cesarianas.

\section{REFERÊNCIAS}

1. Moreira ML, Dutilh Novaes HM. [Hospital admissions in the hospital health care system, SUS and non-SUS: Brazil, 2006]. Rev. Bras. Epidemiol. 2011;14(3):411-22. Portuguese. http://dx.doi.org/10.1590/S1415-790X2011000300006

2. Orange FAd, Passini-Jr R, Melo ASO, Katz L, Coutinho IC, Amorim MMR. Combined spinal-epidural anesthesia and non-pharmacological methods of pain relief during normal childbirth and maternal satisfaction: a randomized clinical trial. Rev. Assoc. Med. Bras. 2012;58(1):112-7. http://dx.doi.org/10.1590/S0104-42302012000100023

3. Donabedian A. The quality of care. How can it be assessed? JAMA. 1988;260(12):1743-8. https://www.ncbi.nlm.nih.gov/pubmed/3045356

4. Wilcock A, Kobayashi L, Murray I. Twenty-five years of obstetric patient satisfaction in North America: a review of the literature. J. Perinat. Neonatal. Nurs. 1997;10(4):36-47. https://www.ncbi.nlm.nih.gov/pubmed/9214962 
5. Hodnett ED. Pain and women's satisfaction with the experience of childbirth: a systematic review. Am. J. Obstet. Gynecol. 2002;186(5 Suppl Nature):S160-72. https://www.ncbi.nlm.nih.gov/pubmed/12011880

6. Domingues RMSM, Santos EMd, Leal MdC. [Aspects of women's satisfaction with childbirth care in a maternity hospital in Rio de Janeiro]. Cad. Saúde Pública. 2004;20(1):S52-S62. Portuguese. http://dx.doi.org/10.1590/S0102-311X2004000700006

7. Shorten A, Shorten B. The importance of mode of birth after previous cesarean: success, satisfaction, and postnatal health. J. Midwifery Womens Health. 2012;57(2):126-32. https://www.ncbi.nlm.nih.gov/pubmed/22432483

8. Dunn EA, O'Herlihy C. Comparison of maternal satisfaction following vaginal delivery after caesarean section and caesarean section after previous vaginal delivery. Eur. J. Obstet. Gynecol. Reprod. Biol. 2005;121(1):56-60.

https://www.ncbi.nlm.nih.gov/pubmed/15950369

9. Garcia Cicuto A, Leite Belisário CR, Barco Tavares B. Puerperal women's satisfaction with their delivery. Invest. Educ. Enferm. 2012;30:208-14.

http://www.redalyc.org/pdf/1052/105224306005.pdf

10. Fundo das Nações Unidas para a Infância (Unicef). The State of the World's Children 2012. Children in an Urban World. New York. 2012. https://www.unicef.org/sowc2012/

11. Paris GF, Monteschio LVC, Oliveira RRd, Latorre MdRDdO, Pelloso SM, Mathias TAdF. [Time trend of the rates of cesarean and vaginal delivery according to the source of financing]. Rev. Bras. Gincecol. Obstet. 2014;36(12):548-54. Portuguese. http://dx.doi.org/10.1590/So100-720320140005038

12. Marcolin AC. [Until when will Brazil be known as the country of cesarean section?] Rev. Bras. Gincecol. Obstet. 2014;36(7):283-9. Portuguese. http://dx.doi.org/10.1590/SO100-720320140005087

13. Domingues RMSM, Dias MAB, Nakamura-Pereira M, Torres JA, d'Orsi E, Pereira APE, et al. Processo de decisão pelo tipo de parto no Brasil: da preferência inicial das mulheres à via de parto final. Cad. Saúde Pública. 2014;30(1):S101-S16.

http://dx.doi.org/10.1590/0102-311X00105113

14. Fonseca J, Martins G. Curso de estatística. 5th ed. São Paulo: Atlas; 1994.

15. Associação Brasileira de Empresas de Pesquisa. Critério Padrão de Classificação Econômica Brasil 2015 (versão preliminar). 2014. http://www.abep.org/criterio-brasil

16. Tabachnick BG, Fidell LS. Using Multivariate Statistics. 4th ed. Boston: Allyn and Bacon; 2001. http://trove.nla.gov.au/version/8267663

17. Conover WJ. Practical Nonparametric Statistics. 3th ed. New York: John Wiley \& Sons; 1999.

18. Hatcher L. A Step-by-Step Approach to Using the SAS System for Factor Analysis and Structural Equation Modeling. Cary, NC: SAS Institute Inc; 1994.

19. Rattray J, Jones MC. Essential elements of questionnaire design and development. JCN. 2007;16(2):234-43. https://www.ncbi.nlm.nih.gov/pubmed/17239058 
20. Pereira JCR. Análise de dados qualitativos: estratégias metodológicas para as ciências da saúde, humanas e sociais. 3rd ed. São Paulo: Editora da Universidade de São Paulo; 2004.

21. Urbina S. Essentials of Psychological Testing. Hoboken, New Jersey: John Wiley \& Sons, Inc.; 2004.

22. Roncone A, Perdichizzi F, Pires OC. [Pain and satisfaction during primiparous labor: parturient and obstetrician view]. Rev. Dor. 2010;11(4):277-81. Portuguese. http://files.bvs.br/upload/S/1806-0013/2010/v11n4/a1647.pdf

23. Consonni EB. Repercussões de um Programa Multidisciplinar de Preparo para o Parto e Maternidade: Ansiedade Materna e Resultados Perinatais. Rev. Bras. Gincecol. Obstet. 2002;24(3):205-205. http://dx.doi.org/10.1590/S0100-72032002000300010

24. Boudreaux ED, O'Hea EL. Patient satisfaction in the Emergency Department: a review of the literature and implications for practice. J. Emerg. Med. 2004;26(1):13-26.

https://www.ncbi.nlm.nih.gov/pubmed/14751474

25. van de Glind I, de Roode S, Goossensen A. Do patients in hospitals benefit from single rooms? A literature review. Health Policy. 2007;84(2-3):153-61.

https://www.ncbi.nlm.nih.gov/pubmed/17631979

26. Brooten D, Roncoli M, Finkler S, Arnold L, Cohen A, Mennuti M. A randomized trial of early hospital discharge and home follow-up of women having cesarean birth. Obstet.

Gynecol. 1994;84(5):832-8. https://www.ncbi.nlm.nih.gov/pubmed/7936522

27. Thurston NE, Dundas JB. Evaluation of an early post partum discharge program. Can. J. Public Health. 1985;76:384-7. https://www.ncbi.nlm.nih.gov/pubmed/4092180

28. Figueiró-Filho EA, Lopes AHA, Senefonte FRdA, Souza Júnior VGd, Botelho CA, Duarte G. [Maternal phenylketonuria: a case report]. Rev. Bras. Gincecol. Obstet. 2004;26(10):813-7. Portuguese. http://dx.doi.org/10.1590/S0100-72032004001000009

29. Silva MBGMd, Lacerda MR. [Newborn screening program: Why to collect in high the hospital one?]. REE. 2003;5(2):50-4. Portuguese.

https://www.fen.ufg.br/fen revista/revista5 2/pdf/pezinho.pdf

30. Sitzia J, Wood N. Patient satisfaction: a review of issues and concepts. Soc. Sci. Med. 1997;45(12):1829-43. https://www.ncbi.nlm.nih.gov/pubmed/9447632

31. Hall JA, Dornan MC. Patient sociodemographic characteristics as predictors of satisfaction with medical care: A meta-analysis. Soc. Sci. Med. 1990;30(7):811-8. https://www.ncbi.nlm.nih.gov/pubmed/2138357

32. d'Orsi E, Brüggemann OM, Diniz CSG, Aguiar JMd, Gusman CR, Torres JA, et al. Desigualdades sociais e satisfação das mulheres com o atendimento ao parto no Brasil: estudo nacional de base hospitalar. Cad. Saúde Pública. 2014;30:S154-S68. http://www.scielosp.org/pdf/csp/v30s1/0102-311X-csp-30-s1-0154.pdf

33. Benute GRG, Nomura RY, Santos AMd, Zarvos MA, Lucia MCSd, Francisco RPV. [Preference in the process of parturition: a comparison between primiparous and nulliparous women]. Rev. Bras. Gincecol. Obstet. 2013;35(6):281-5. Portuguese. http://dx.doi.org/10.1590/S0100-72032013000600008 
34. Carniel EdF, Zanolli MdL, Morcillo AM. [Risk factors for the indication of caesarean section in Campinas (SP)]. Rev. Bras. Gincecol. Obstet. 2007;29:34-40. Portuguese. http://dx.doi.org/10.1590/S0100-72032007000100006

35. Armigliato ME, Prado DGdA, Melo TMd, Martinez MANdS, Lopes AC, Amantini RCB, et al. [Hearing health service evaluation under the perspective of the users: proposal of an instrument]. Rev. Soc. Bras. Fonoaudiol. 2010;15:32-9. Portuguese.

http://dx.doi.org/10.1590/S1516-80342010000100008

Recebido: 15/12/2016; Aceito e publicado: 17/01/2017

Correspondência: Prof ${ }^{\mathrm{a}} \mathrm{Dr}^{\mathrm{a}}$ Angela Maria Bacha. Endereço: Caism - Rua Alexander Fleming, 101 - Cidade Universitária Zeferino Vaz - Distrito de Barão Geraldo - CEP 13083-881. Email: angela.bacha@reitoria.unicamp.br Telefone: 19-3521-2940.

Local do estudo: Hospital da Mulher Prof. Dr. José Aristodemo Pinotti - Caism/Unicamp, Campinas (SP), Brasil.

Financiamento: os custos inerentes à realização do presente estudo foram cobertos pelo Hospital da Mulher Prof. Dr. José Aristodemo Pinotti - Caism e pelo programa de Bolsa Auxílio Social do Serviço de Apoio do Estudante da Unicamp.

Conflito de Interesses: os autores não declararam conflito de interesses

(c) This is an Open Access article distributed under the terms of the Creative Commons Attribution License, which permits unrestricted use, distribution, and reproduction in any medium, provided the original work is properly cited. 